Original article

\title{
Metastasis suppressor proteins in cutaneous squamous cell carcinoma
}

\author{
Onder Bozdogan ${ }^{\mathrm{a}}$, Ibrahim Vargel ${ }^{\mathrm{b}}$, Tarik Cavusoglu ${ }^{\mathrm{c}}$, Ayse A. Karabulut ${ }^{\mathrm{d}}$, \\ Gurbet Karahan ${ }^{\mathrm{e}}$, Nilufer Sayar ${ }^{\mathrm{f}}$, Pinar Atasoy ${ }^{\mathrm{g}}$, Isik G. Yulug ${ }^{\mathrm{e}, *}$ \\ a Ankara Numune Education and Research Hospital, Department of Pathology, Ankara, Turkey \\ ${ }^{\mathrm{b}}$ Hacettepe University, Medical Faculty, Department of Plastic Surgery, Science Institute, Department of Bioengineering, Ankara, Turkey \\ c Private Practice, Plastic Surgery, Ankara, Turkey \\ ${ }^{\mathrm{d}}$ Kırıkkale University, Faculty of Medicine, Department of Dermatology, Kirıkkale, Turkey \\ e Bilkent University, Faculty of Science, Department of Molecular Biology and Genetics, Ankara, Turkey \\ ${ }^{\mathrm{f}}$ Istanbul Medipol University, International School of Medicine, Department of Physiology, Istanbul, Turkey \\ ${ }^{g}$ Kirlkkale University, Faculty of Medicine, Department of Pathology, Kirkkale, Turkey
}

\section{A R T I C L E I N F O}

\section{Article history:}

Received 9 August 2015

Received in revised form

15 November 2015

Accepted 3 December 2015

\section{Keywords:}

Skin

Squamous cell carcinoma

Metastasis suppressor proteins

A-431

$\mathrm{HaCaT}$

\begin{abstract}
A B S T R A C T
Cutaneous squamous cell carcinomas (cSCCs) are common human carcinomas. Despite having metastasizing capacities, they usually show less aggressive progression compared to squamous cell carcinoma (SCC) of other organs. Metastasis suppressor proteins (MSPs) are a group of proteins that control and slow-down the metastatic process. In this study, we established the importance of seven well-defined MSPs including NDRG1, NM23-H1, RhoGDI2, E-cadherin, CD82/KAI1, MKK4, and AKAP12 in cSCCs.

Protein expression levels of the selected MSPs were detected in 32 cSCCs, 6 in situ SCCs, and two skin cell lines (HaCaT, A-431) by immunohistochemistry. The results were evaluated semi-quantitatively using the HSCORE system. In addition, mRNA expression levels were detected by qRT-PCR in the cell lines.

The HSCOREs of NM23-H1 were similar in cSCCs and normal skin tissues, while RGHOGDI2, E-cadherin and AKAP12 were significantly downregulated in CSCCs compared to normal skin. The levels of MKK4, NDRG1 and CD82 were partially conserved in cSCCs. In stage ISCCs, nuclear staining of NM23-H1 (NM23H1 nuc) was significantly lower than in stage II/III SCCs. Only nuclear staining of MKK4 (MKK4nuc) showed significantly higher scores in in situ carcinomas compared to invasive SCCs.

In conclusion, similar to other human tumors, we have demonstrated complex differential expression patterns for the MSPs in in-situ and invasive cSCCs. This complex MSP signature warrants further biological and experimental pathway research.
\end{abstract}

(C) 2016 Elsevier GmbH. All rights reserved.

\section{Introduction}

Non-melanoma skin cancers (NMSC) are the most common human malignant neoplasms, and create significant medical, economical, and social problems for the healthcare services worldwide [1-3]. Although there are other types of NMSC, this term commonly refers to the two common neoplasms of cutaneous squamous cell carcinoma (cSCC) and basal cell carcinoma (BCC) [1]. BCCs are slow growing, malignant, yet rarely metastasizing carcinomas [4]. On the contrary, CSCCs are more aggressive in behavior, and have considerably higher metastatic capacities than BCCs [4].

Metastasis is a very complex and multistep biological process directed by various proteins and pathways [5]. Though various

\footnotetext{
* Corresponding author at: Bilkent University, Faculty of Science, Department of Molecular Biology and Genetics, TR-06800 Ankara, Turkey.

E-mail address: yulug@fen.bilkent.edu.tr (I.G. Yulug).
}

proteins support metastasis, a group of proteins called metastasis suppressor proteins (MSPs) specifically inhibit or slow metastasis $[6,7]$. As a definition, pure MSPs should only suppress metastasis without any effect on tumorigenicity (e.g. proliferation). However, in the complex environment of a cell, they usually have other important properties as tumor suppressor activities, affecting carcinogenesis, besides these MSP functions [6]. cSCCs differ from internal identical organ cancers in that they have lower metastatic rates and result in better prognosis [4]. Thus, cSCCs are interesting biological models for the research of metastasis suppressors. To establish the importance of the MSPs in non-melanoma skin cancer, we selected seven essential and well-defined MSPs (NDRG1, NM23-H1, RhoGDI2, E-cadherin, CD82/KAI1, MKK4, and AKAP12) that affect different steps of metastasis.

The main aim of this study was to analyze the expression patterns of these seven important MSPs that may contribute to the inhibition of metastasis pathways in cSCCs, as well as in squamous cell lines. We also established the association between 
these proteins and important clinicopathological parameters in cSCC.

\section{Materials and methods}

\subsection{Study groups}

A total of 38 SCCs composed of 32 tissue samples of classical squamous cell carcinoma (SCC-NOS) of the skin and 6 in situ carcinoma tissues, obtained from 37 patients $(26 \mathrm{M} / 11 \mathrm{~F})$, were included in this study. All patients were Caucasians, and the detailed characteristics of the study group are summarized in Appendix 1. SCCs were graded by four-tiered system as well (Grade 1), moderately (Grade 2 ) poorly differentiated (Grade 3 ) and anaplastic or undifferentiated tumors (Grade 4) [8]. Data for the SCCs were collected by using the CAP (The College of American Pathologists) protocols for squamous carcinoma of the skin (www.cap.org).

\subsection{Normal skin control group}

Ten normal skin tissues ( $4 \mathrm{M} / 6 \mathrm{~F})$ from reconstructive operations, confirmed as normal by microscopy, were included as the normal tissue group. The normal group included skin tissues from different localizations; two from the face, three from the extremities, three from the breast, and two from the abdomen. This control group was the same as the one used in another previous study of our group [9]. Normal, non-lesional epidermis adjacent to SCC (N-SCC) was also integrated in this study.

\subsection{Cell lines}

The normal immortalized keratinocyte cell line (HaCaT) and the vulvar squamous carcinoma cell line (A-431) were used for immunohistochemistry and quantitative reverse transcriptase-PCR (qRT-PCR) studies [10,11].

\subsection{Immunohistochemistry technique and analysis}

The immunostaining procedure was performed by the classical labeled streptavidin-biotin immuno-enzymatic antigen detection system (UltraVision-Thermo Scientific; Waltham, MA, USA) with DAB chromogen, in the Thermo-Shandon Sequenza ${ }^{\circledR}$ manual staining station (Waltham, MA, USA). The primary antibody step was skipped for the negative control. The sources of primary antibodies and the technical details are demonstrated in Appendix 2.

All immunohistochemically stained slides were evaluated by external and internal controls. Stained slides were semiquantitatively evaluated using a specific immunohistochemical histological score technique, (HSCORE), with minor modifications. The analysis technique was described in detail in the literature and in our previous study $[9,12]$.

\subsection{Cell culture}

The A-431 cell line was cultured in Dulbecco's Minimal Essential Medium (DMEM-low glucose, Hyclone-Thermo Scientific, Waltham, MA, USA), and HaCaT cell line was grown in DMEMHigh glucose (Hyclone), supplemented with $10 \%$ fetal bovine serum (FBS) and $50 \mathrm{mg} / \mathrm{ml}$ penicillin/streptomycin, at $37^{\circ} \mathrm{C}$, and in $5 \%$ carbon dioxide.

\subsection{Real-time PCR ( $q R T-P C R)$}

500 ng of total RNA was reverse-transcribed using oligo-dT primers with the First Strand cDNA Synthesis Kit (Fermentas,
Thermo, Waltham, MA, USA). All qRT-PCR experiments were performed using the $\mathrm{SYBR}^{\circledR}$ Green reagent (Thermo) in an MX3005P thermocycler (Strategene ${ }^{\circledR}$, Agilent, Santa Clara, CA, USA). The GAPDH (glyceraldehyde-3-phosphate dehydrogenase) and HPRT1 (hypoxanthine phosphoribosyl transferase 1) reference genes were selected for normalization. Data was analyzed using the free REST ${ }^{\odot}$ 2009 software (Qiagen, Hilden, Germany) [13]. The sequences of primers used are accesible from our previously published paper [9].

\subsection{Statistical analysis}

Statistical analyses were performed using the PASW ${ }^{\circledR}$ Statistics 18 software (Chicago, IL, USA). The differences between the HSCOREs of the groups were first analyzed with the non-parametric Kruskal-Wallis one-way analysis test; followed by Mann-Whitney $U$ test was applied. $p \leq 0.05$ was accepted as significant. The "Bonferroni correction" was used to reduce type I errors. The correlation between the parameters was analyzed by Spearman's correlation test, where $r \geq 0.25$ and $p \leq 0.05$ were accepted as significant.

\subsection{Ethics statement}

This study was financially supported by the Scientific and Technical Research Council of Turkey (TUBITAK, grant number SBAG-108S184). The project was approved by the Kırıkkale University Local Ethics Committee (07.04.2008/2008-039).

\section{Results}

\subsection{Immunohistochemical staining results}

\subsubsection{Tissue study}

Cytoplasmic positivity of NM23-H1 (NM23-H1 $1_{\text {cyt }}$ ) and NDRG1 (NDRG1 $1_{\text {cyt }}$ ) was detected to be strong and homogeneous in all of the in situ and invasive cSCCs (Fig. 1A, B, F, G). However, nuclear

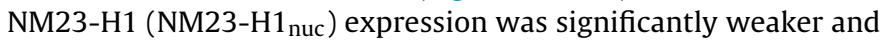
was detected in only two of in situ SCCs (IS-SCC, 33.3\%) and 15 cSCCs (46.8\%) compared to normal skin. Nuclear positivity of NDRG1 (NDRG $1_{\text {nuc }}$ ) was seen in 4 of 6 IS-SCCs $(66.6 \%)$, and 29 of 32 cSCCs (90.6\%).

E-cadherin, CD82 and AKAP12 proteins show only weak and medium cytoplasmic-membranous staining (Figs. 2A, B, F, G and $3 \mathrm{~A}, \mathrm{~B})$. E-cadherin and CD82 positivity was detected in all of IS-SCCs, but AKAP12 positivity was observed in 4 IS-SCCs (66.6\%). E-cadherin, CD82, and AKAP12 expressions were detected in $84.3 \%$, $68.7 \%$ and $40.6 \%$ of the cSCCs, respectively.

RHOGDI2 showed cytoplasmic positivity in 5 (83.3\%) and 30 (93.7\%) of the in situ and invasive cases, respectively, though with weak to medium strength only (Fig. 3F, G). Nuclear positivity of RHOGDI2 (RHOGDI2 $2_{\text {nuc }}$ ) was weaker, yet more heterogeneous, in 4 of 6 IS-SCCs (66.6\%) and 14 of 32 cSCCs (43.7\%).

MKK $4_{\text {cyt }}$ was detected in all of IS-SCCs, and weak MKK4 $4_{\text {nuc }}$ was detected in 5 of 6 IS-SCCs (83.3\%) (Fig. 4A). MKK4 immunostaining of cSCCs showed weak to medium cytoplasmic positivity in 25 cases (78.1\%), and weak nuclear positivity in 7 cSCCs (21.8\%) (Fig. 4B).

\subsubsection{Cell lines}

NM23-H1 showed strong cytoplasmic positivity in the both HaCaT and A-431 cell lines (Fig. 1C, D). However, focal nuclear positivity was stronger in the HaCaT cell line. Significant positivity was observed with NDRG1 antibody in both of the cell lines (Fig. 1H, I). E-cadherin expression was stronger in the HaCaT cell line compared to A-431 (Fig. 2C, D). CD82/KAI1 showed medium levels of positivity in both cell lines (Fig. 2H, I). RHOGDI2 showed strong but heterogeneous positivity in the $\mathrm{HaCaT}$ and $\mathrm{A}-431$ cell lines (Fig. $3 \mathrm{H}$, 


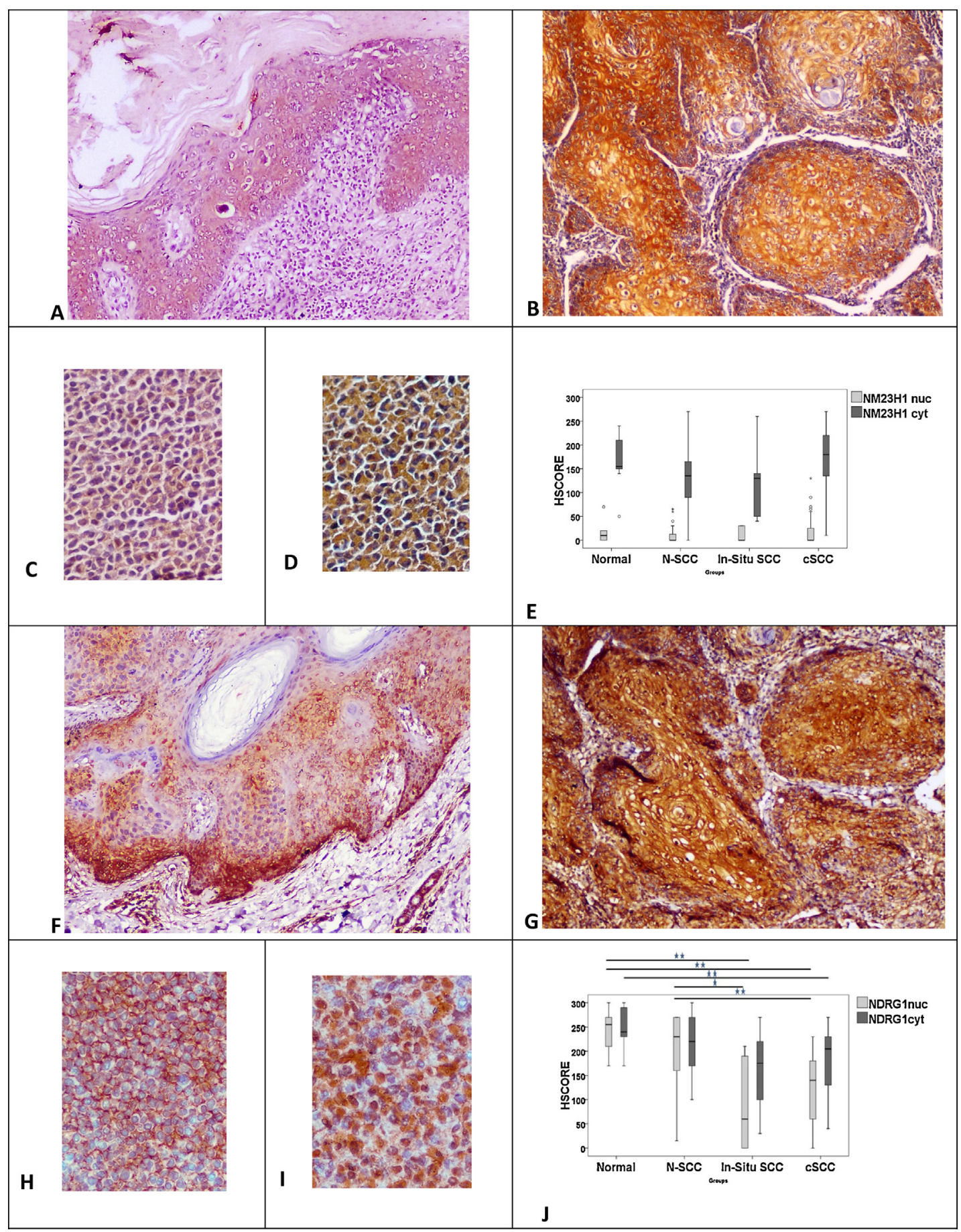

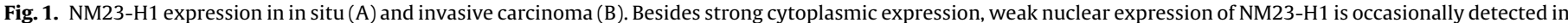

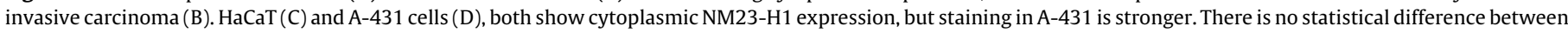

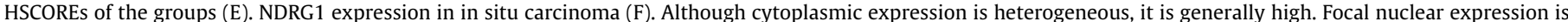

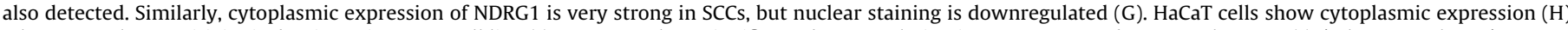

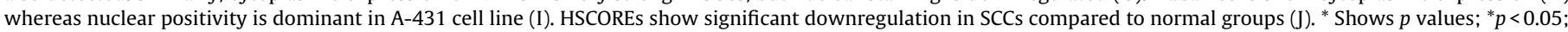
${ }^{* *} p<0.01$. Original magnification: A; B; F; G $\times 100, C$; D; H; I $\times 200$.

I). AKAP12 and MKK4 staining was either very weak or completely lost in both cell lines (Figs. 3C, D and 4C, D).

\subsubsection{Comparative statistics}

When HSCOREs of normal epidermis $(\mathrm{N})$ were compared to in situ carcinomas, E-cadherin $(p=0.016)$, RHOGDI2 ${ }_{\text {nuc }}(p=0.002)$, and NDRG1 $1_{\text {nuc }}(p=0.003)$ stainings showed significantly lower scores in in situ carcinomas. However, when compared to normal epidermis adjacent to SCC (N-SCC), only HSCOREs of NDRG $1_{\text {nuc }}$ were significantly lower in in situ carcinomas $(p=0.011)$.

NDRG1 $1_{\text {nuc/cyt }} \quad(p=0.001 / p=0.007), \quad$ E-cadherin $\quad(p=0.001)$, RHOGDI $2_{\text {nuc } / \text { cyt }}(p=0.001 / p=0.001), \operatorname{MKK} 4$ nuc $(p=0.001)$, and AKAP12 $(p=0.001)$ expressions were decreased in CSCC compared to N. When HSCOREs of N-SCC were compared to cSCCs, 


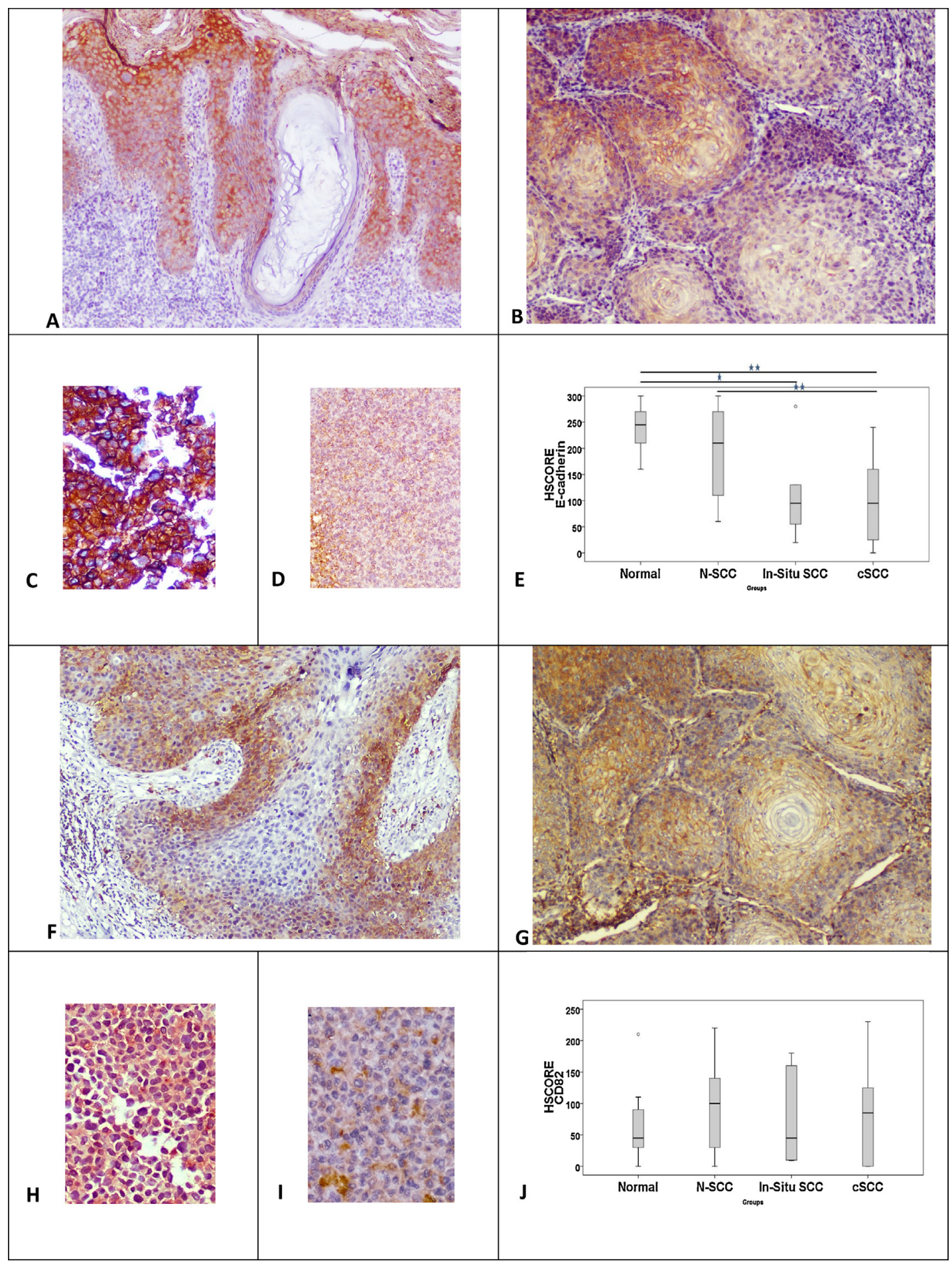

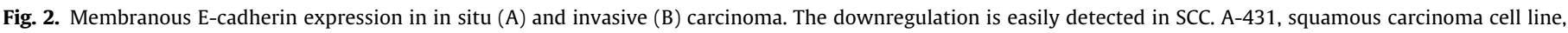

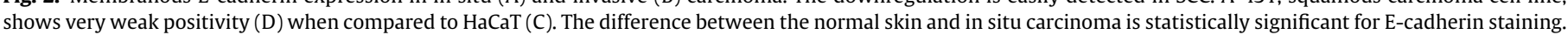

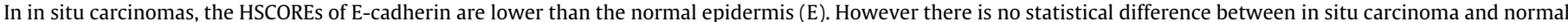

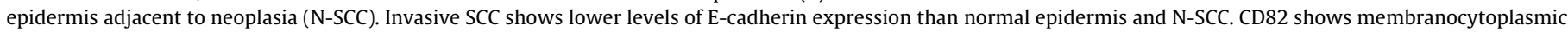

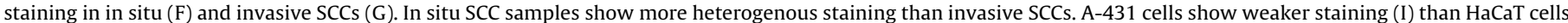

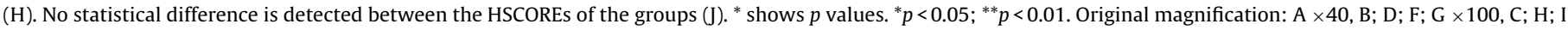
$\times 200$.

NDRG1 $1_{\text {nuc }}(p=0.001)$, E-cadherin $(p=0.001)$, and RHOGDI $2_{\text {nuc } / \text { cyt }}$ $(p=0.001 / p=0.001)$ showed significantly lower scores in cSCCs.

NM23-H1 nuc showed significantly lower scores in stage I cSCCs than stage II/III SCCs $(p=0.049)$. MKK4 $4_{\text {nuc }}$ was the only one that showed higher scores in in situ carcinomas when compared to invasive SCCs $(p=0.011)$.

\subsubsection{Correlation analysis}

There were limited numbers of positive significant correlations $(p=0.01$ level) between several markers as follows: E-cadherin-RHOGDI2 $2_{\text {cyt }} \quad(r=0.452), \quad$ RHOGDI $2_{\text {cyt }}-$ MKK $4_{\text {cyt }}$ $(r=0.486)$, and $\mathrm{MKK}_{\text {nuc }}-\mathrm{MKK} 4_{\text {cyt }} \quad(r=0.481)$ in invasive SCCs. 


\subsubsection{Real time PCR results}

NM23-H1 and E-cadherin expression levels did not differ between $\mathrm{A}-431$ and $\mathrm{HaCaT}$ cell lines. Yet, NDRG1 expression was upregulated (34.4-fold, $p=0.001$ ), whereas ARHGDIB (RHOGDI2, -4.7-fold, $p=0.001$ ), MKK4 ( -2.1 -fold, $p=0.001$ ), CD82/KAI ( -2.4 fold, $p=0.001)$ and $A K A P 12$ ( -9.7 -fold, $p=0.001)$ expressions were downregulated in A-431 cells compared to HaCat cells.

\section{Discussion}

In this study, we demonstrated different expression patterns of MSPs in cutaneous SCCs. NM23-H1 levels were conserved in in situ and invasive carcinomas, and in squamous carcinoma cell lines. Although the role of NM23-H1 in metastasis suppression is well described in other carcinomas, it is not clear in NMSC [6,7]. Similar

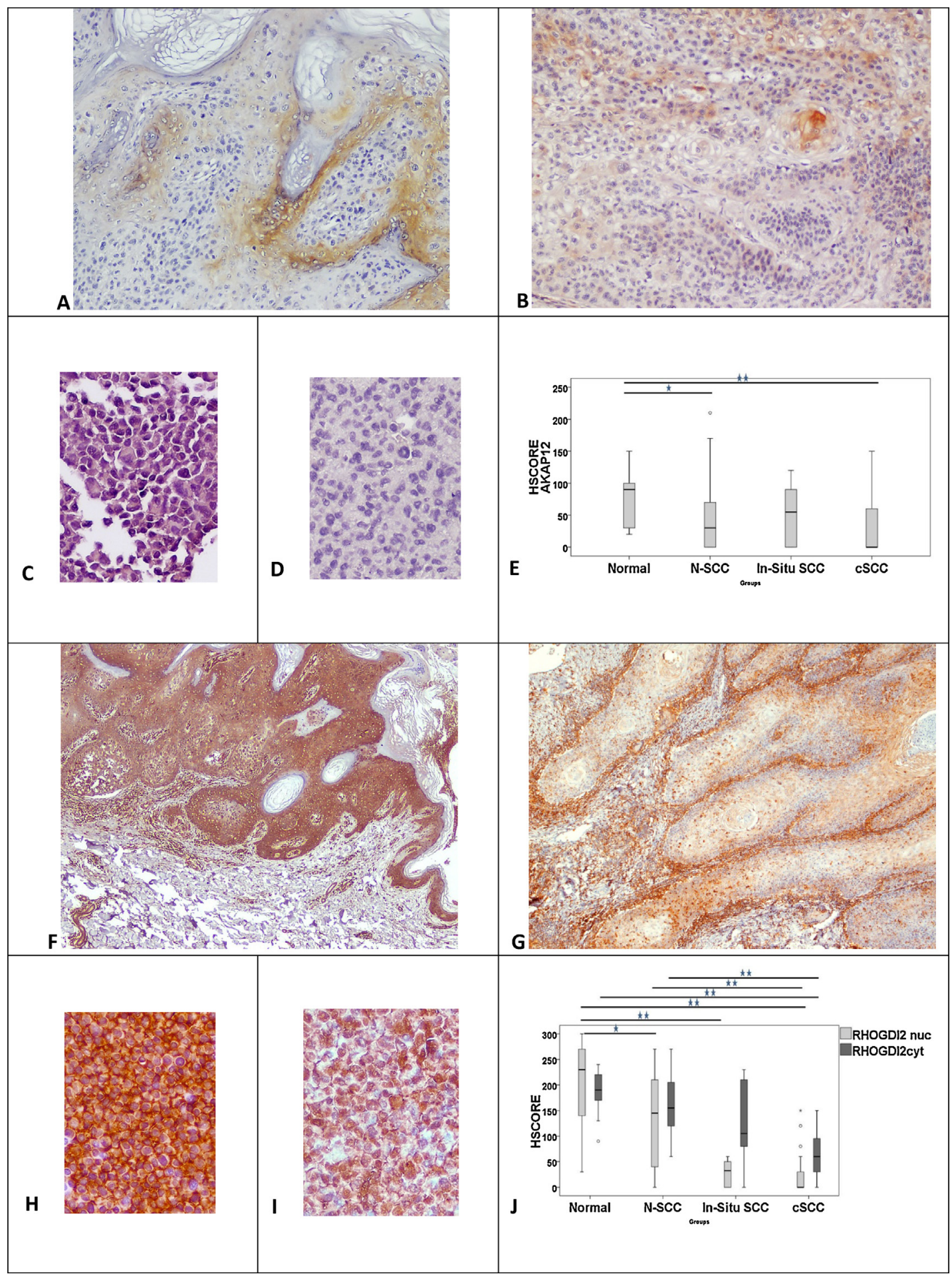

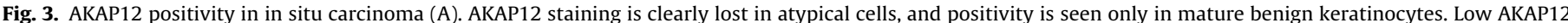

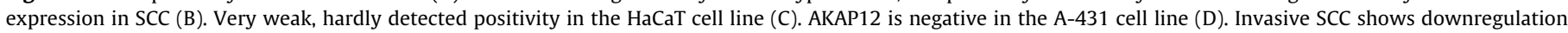

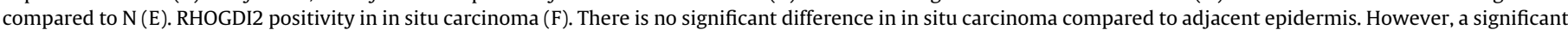

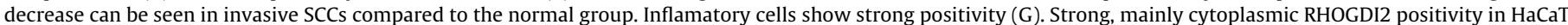

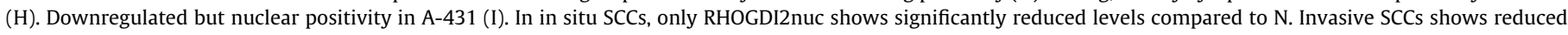
levels of RHOGDI2nuc/cyt compared to both N and N-SCC (E). * shows $p$ value. ${ }^{*} p \leq 0.05 ;{ }^{* *} p \leq 0.01$. Original Magnification: Fx 40, A; B; G $\times 100$, D; E; H; I $\times 200$. 


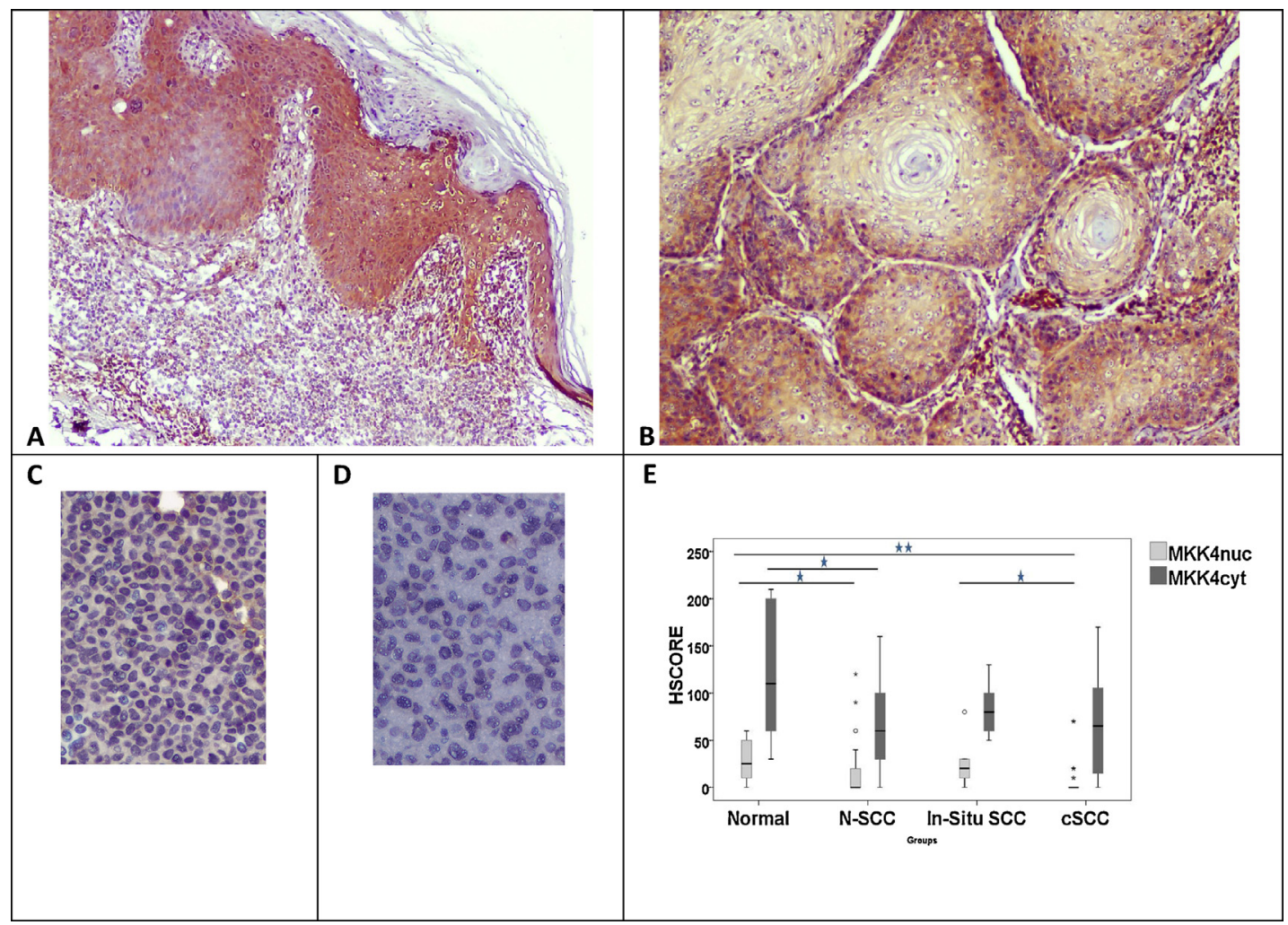

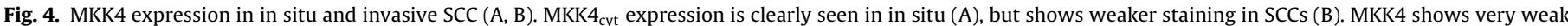

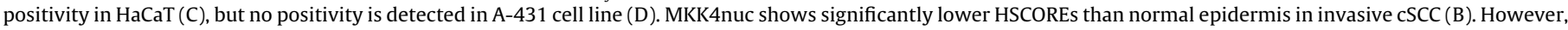

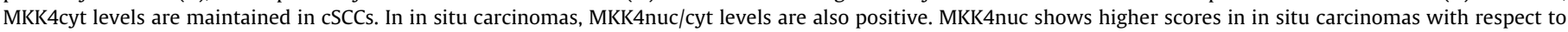
invasive SCCs. ${ }^{*}$ shows $p$ value. ${ }^{*} p<0.05 ;{ }^{* *} p<0.01$. Original magnification: A $\times 40, B \times 100, C ; D \times 200$.

to our findings, Ro et al. [14] and Stephenson et al. [15] showed that the expression of NM23-H1 was well conserved in cSCCs of the skin and keratoacanthomas (KA). However, Kanitakis et al. [16] obtained contradictory results and showed reduced NM23-H1 levels in cSCCs. The sustained NM23-H1 levels in cSCCs probably contribute to their non-metastasizing features as observed in other carcinomas.

Despite being downregulated in carcinoma samples compared to normal tissues, the cytoplasmic NDRG1 expression is generally maintained in in situ and invasive SCCs. Furthermore, mRNA expression of NDRG1 in the SCC cell line, A-431, was 34-fold increased compared to normal immortalized cell line, HaCaT. In accordance with our findings, Dang et al. [17] demonstrated that NDRG1 mRNA expression increased 5.87-fold in actinic keratoses and 7.07-fold in cSCCs when compared to normal skin. The importance of NDRG1 expression has been examined in SCCs of the internal organs, in the literature. Dos Santos et al. [18] showed significant upregulation of NDRG1 in oral and oropharyngeal SCCs, and concluded that NDRG1 overexpression was significantly correlated with long-term survival. Similar results were also reported by Chang et al. [19]. The prognostic importance of NDRG1 was also demonstrated in esophageal SCCs, as well as in other human tumors, such as tumors of prostate, breast, and colon [20-24]. However, the MSP function of NDRG1 is probably carcinomadependent. NDRG1 overexpression in hepatocellular carcinoma has been shown to be an indicator of poor prognosis [25]. In non small cell lung carcinoma, its expression is significantly associated with advanced stages and weak vascularization [26].

Although HSCORE values of CD82 in in situ and invasive squamous carcinoma were not different from normal skin, only $68.7 \%$ of cSCCs showed CD82 positivity, while limited downregulation of mRNA expression (2.4-fold) was detected in the A-431 cell line.
Similar to our findings, Okochi et al. [27] showed significant downregulation of CD82 in basal cell carcinoma of the skin, despite conserved expression in Bowen disease. The findings regarding CD82 expression in other types of human SCC are controversial. CD82 downregulation has been demonstrated in oral, cervical, penile, laryngeal, head and neck, and lung SCCs [28-30]. Interestingly, when compared to cSCCs, all of these carcinomas possess greater metastasis potential. Relatively conserved levels of CD82 may support the non-metastasizing features of cutaneous SCCs.

Cytoplasmic MKK4 expression was maintained at relatively higher levels in SCCs in our study. MKK4 expression was previously shown to be reduced during cancer progression in prostate and ovary carcinomas $[31,32]$. Similar results were shown in other epithelial cancers, including endometrial carcinoma, gastric carcinoma, and pancreatic cancer [33-35]. MKK4 is generally accepted to be a metastasis suppressor, but there are still conflicting reports in the literature. Huang et al. found higher expression of MKK4 in laryngeal SCCs in comparison with their normal counterparts, in addition to a positive correlation between higher expression of MKK4 and increased metastasis [36]. In a recent experimental article, Finegan et al. [37] proposed that MKK4 has pro-oncogenic roles in skin carcinoma. Our data may support their hypothesis in cSCCs.

The HSCOREs of RHOGDI2 were significantly downregulated in cSCCs. However, RHOGDI2 cytoplasmic expression was maintained in in situ carcinoma. RHOGDI2 expression has not been studied in skin carcinomas previously as far as we are aware. The MSP function of RHOGDI2 was first shown to be a metastasis suppressor gene in bladder carcinoma [38]. Because of conflicting reports, the metastasis suppressor function of this protein could be considered to be tissue or organ-dependent. RHOGDI2 has been previously shown to be upregulated in gastric and ovarian carcinoma, and probably has a dual role in carcinogenesis [39]. 
E-cadherin levels were significantly downregulated in cSCCs. Downregulation of E-cadherin levels has been well demonstrated in cSCC before [40-42]. However, we found that mRNA levels were not changed in the squamous cell carcinoma cell line. It has also been reported that a reduced level of E-cadherin expression was more evident in acantholytic subtypes of cSCCs [41].

We demonstrated that AKAP12 was downregulated in cSCC, with less than half of the cSCCs being positive for AKAP12 staining. Significant mRNA downregulation of AKAP12 was also detected in the A-431 SCC cell line. The decrease in AKAP12 levels in human carcinomas has been reported in various types of human cancers [43]. Yet, the expression pattern of AKAP12 is not known in skin carcinomas. A recent study focused on epigenetic regulation of AKAP12 in skin cancer and pointed out that the promoter methylation frequencies were significantly higher in carcinomas with respect to normal skin tissues. AKAP12 promoter methylation frequencies were demonstrated to be $89.6 \%, 87.1 \%$ and $51.2 \%$ in cSCC, BCC and AK, respectively [44].

In this study, NM23-H1, NDRG1, RHOGDI2 and MKK4 antibodies showed both cytoplasmic and nuclear positivity. It is well known that the subcellular localization of the proteins alters their functions [45]. The importance of this fact is not clear in MSPs. However, Epstein-Barr virus nuclear antigen 3C (EBNA-3C) promotes Nm23$\mathrm{H} 1$ nuclear localization and modulate Nm23-H1 activities [46]. Ismail et al. [47] showed that nuclear localization of NM23-H1 protein in breast cancer is seen more frequently in node-positive metastatic cases. They concluded that abnormal nuclear accumulation of the protein may indicate tumor progression [47]. Similarly, we demonstrated that nuclear NM23-H1 was significantly positively correlated with stage. The nuclear sequestration of NM23-H1 may reduce or alter the normal function of the protein. Besides NM23-H1, the importance of subcellular localization of NDRG1 has also been emphasized in the literature [48-50]. Although nuclear positivity of MKK4 and RHOGDI has been demonstrated in the literature, to our knowledge, its meaning is not well known [35,51].

\section{Conclusions}

Although NM23-H1 expression was maintained at constant levels, RGHOGDI2, E-cadherin, and AKAP12 were significantly downregulated in cSCCs. Furthermore, MKK4, NDRG1 and CD82 were also partially expressed in cSCCs. Similar to other human tumors, cSCCs show a distinct MSP profile. Data from this study might also reveal possible pathways among MSPs, when combined with the current knowledge on related pathways. This relationship between these MSPs warrants further biological and experimental pathway research to better understand the metastatic events with regard to SCCs.

\section{Acknowledgements}

This study was financially supported by the Scientific and Technical Research Council of Turkey (TUBITAK, grant number SBAG-108S184). The project was approved by the Kırıkkale University Local Ethics Committee (07.04.2008/2008-039).

\section{Appendix A. Supplementary data}

Supplementary data associated with this article can be found, in the online version, at http://dx.doi.org/10.1016/j.prp.2015.12.018.

\section{References}

[1] A. Lomas, J. Leonardi-Bee, F. Bath-Hextall, A systematic review of worldwide incidence of nonmelanoma skin cancer, Br. J. Dermatol. 166 (5) (2012) 1069-1080.
[2] V. Madan, J.T. Lear, R.M. Szeimies, Non-melanoma skin cancer, Lancet 375 (9715) (2010) 673-685.

[3] T.S. Housman, et al., Skin cancer is among the most costly of all cancers to treat for the Medicare population, J. Am. Acad. Dermatol. 48 (3) (2003) 425-429.

[4] A.S. Weinberg, C.A. Ogle, E.K. Shim, Metastatic cutaneous squamous cell carcinoma: an update, Dermatol. Surg. 33 (8) (2007) 885-899.

[5] S. Acharyya, L. Matrisian, J.M. D.R.W., Invasion and metastasis, in: M.J (Ed.), Molecular Basis of Cancer, Elsevier, Philadelphia, 2015.

[6] L.A. Shevde, D.R. Welch, Metastasis suppressor pathways - an evolving paradigm, Cancer Lett. 198 (1) (2003) 1-20.

[7] C.W. Rinker-Schaeffer, et al., Metastasis suppressor proteins: discovery, molecular mechanisms, and clinical application, Clin. Cancer Res. 12 (13) (2006) 3882-3889.

[8] E. Calonje, et al., Tumors of the surface epithelium, in: T.B. Eduardo Calonje, A.J. Lazar, P.H. McKee (Eds.), Pathology of the Skin with Clinical Correlations, Elsevier, Saunders, Philadeplphia, PA, 2012.

[9] O. Bozdogan, et al., Differential expression patterns of metastasis suppressor proteins in basal cell carcinoma, Int. J. Dermatol. 54 (8) (2015) 905-915.

[10] P. Boukamp, et al., Normal keratinization in a spontaneously immortalized aneuploid human keratinocyte cell line, J. Cell Biol. 106 (3) (1988) 761-771.

[11] K. Pekkola-Heino, et al., Radiation response of vulvar squamous cell carcinoma (UM-SCV-1A, UM-SCV-1B, UM-SCV-2, and A-431) cells in vitro, Cancer Res. 49 (17) (1989) 4876-4878.

[12] D.A. Budwit-Novotny, et al., Immunohistochemical analyses of estrogen receptor in endometrial adenocarcinoma using a monoclonal antibody, Cancer Res. 46 (10) (1986) 5419-5425.

[13] M.W. Pfaffl, G.W. Horgan, L. Dempfle, Relative expression software tool (REST) for group-wise comparison and statistical analysis of relative expression results in real-time PCR, Nucleic Acids Res. 30 (9) (2002) pe36.

[14] Y.S. Ro, S.J. Jeong, Expression of the nucleoside diphosphate kinase in human skin cancers: an immunohistochemical study, J. Korean Med. Sci. 10 (2) (1995) 97-102.

[15] T.J. Stephenson, et al., 'Anti-metastatic' nm23 gene product expression in keratoacanthoma and squamous cell carcinoma, Dermatology 187 (2) (1993) 95-99.

[16] J. Kanitakis, et al., Expression of the nm23 metastasis-suppressor gene product in skin tumors, J. Cutan. Pathol. 24 (3) (1997) 151-156.

[17] C. Dang, et al., Identification of dysregulated genes in cutaneous squamous cell carcinoma, Oncol. Rep. 16 (3) (2006) 513-519.

[18] M. Dos Santos, et al., Prognostic significance of NDRG1 expression in oral and oropharyngeal squamous cell carcinoma, Mol. Biol. Rep. 39 (12) (2012) 10157-10165.

[19] J.T. Chang, et al., Identification of differentially expressed genes in oral squamous cell carcinoma (OSCC): overexpression of NPM, CDK1 and NDRG1 and underexpression of CHES1, Int. J. Cancer 114 (6) (2005) 942-949.

[20] T. Ando, et al., Decreased expression of NDRG1 is correlated with tumor progression and poor prognosis in patients with esophageal squamous cell carcinoma, Dis. Esophagus 19 (6) (2006) 454-458.

[21] S. Bandyopadhyay, et al., The tumor metastasis suppressor gene Drg-1 down-regulates the expression of activating transcription factor 3 in prostate cancer, Cancer Res. 66 (24) (2006) 11983-11990.

[22] S. Bandyopadhyay, et al., Role of the putative tumor metastasis suppressor gene Drg-1 in breast cancer progression, Oncogene 23 (33) (2004) 5675-5681

[23] B. Strzelczyk, et al., Identification of high-risk stage II colorectal tumors by combined analysis of the NDRG1 gene expression and the depth of tumor invasion, Ann. Surg. Oncol. 16 (5) (2009) 1287-1294.

[24] H. Cangul, Hypoxia upregulates the expression of the NDRG1 gene leading to its overexpression in various human cancers, BMC Genet. 5 (2004) p27.

[25] M.S. Chua, et al., Overexpression of NDRG1 is an indicator of poor prognosis in hepatocellular carcinoma, Mod. Pathol. 20 (1) (2007) 76-83.

[26] C. Fan, et al., Increased NDRG1 expression is associated with advanced T stages and poor vascularization in non-small cell lung cancer, Pathol. Oncol. Res. 18 (3) (2012) 549-556.

[27] H. Okochi, et al., Expression of tetra-spans transmembrane family (CD9, CD37 CD53, CD63, CD81 and CD82) in normal and neoplastic human keratinocytes: an association of CD9 with alpha 3 beta 1 integrin, Br. J. Dermatol. 137 (6) (1997) 856-863.

[28] M.E. Buim, et al., Downregulation of CD9 protein expression is associated with aggressive behavior of oral squamous cell carcinoma, Oral Oncol. 46 (3) (2010) 166-171.

[29] Y. Xiong, et al., Expression of metastasis suppressor gene KAI1/CD82 in cervical squamous cell carcinoma and its clinical significance, Ai Zheng 24 (1) (2005) 110-115.

[30] C. Protzel, et al., Down-regulation of the metastasis suppressor protein KAI1/CD82 correlates with occurrence of metastasis, prognosis and presence of HPV DNA in human penile squamous cell carcinoma, Virchows Arch. 452 (4) (2008) 369-375

[31] S.D. Yamada, et al., Mitogen-activated protein kinase kinase 4 (MKK4) acts as a metastasis suppressor gene in human ovarian carcinoma, Cancer Res. 62 (22) (2002) 6717-6723.

[32] H.L. Kim, et al., Mitogen-activated protein kinase kinase 4 metastasis suppressor gene expression is inversely related to histological pattern in advancing human prostatic cancers, Cancer Res. 61 (7) (2001) 2833-2837.

[33] M. Ishikawa, et al., Functional and clinicopathological analysis of loss of MKK4 expression in endometrial cancer, Oncology 79 (3-4) (2010) 238-246. 
[34] S.C. Cunningham, et al., MKK4 status predicts survival after resection of gastric adenocarcinoma, Arch. Surg. 141 (11) (2006) 1095-1099 (discussion 1100).

[35] W. Xin, et al., MAP2K4/MKK4 expression in pancreatic cancer: genetic validation of immunohistochemistry and relationship to disease course, Clin. Cancer Res. 10 (24) (2004) 8516-8520.

[36] C. Huang, et al., Overexpression of mitogen-activated protein kinase kinase 4 and nuclear factor-kappaB in laryngeal squamous cell carcinoma: a potential indicator for poor prognosis, Oncol. Rep. 22 (1) (2009) 89-95.

[37] K.G. Finegan, C. Tournier, The mitogen-activated protein kinase kinase 4 has a pro-oncogenic role in skin cancer, Cancer Res. 70 (14) (2010) 5797-5806.

[38] J.J. Gildea, et al., RhoGDI2 is an invasion and metastasis suppressor gene in human cancer, Cancer Res. 62 (22) (2002) 6418-6423.

[39] E.M. Griner, D. Theodorescu, The faces and friends of RhoGDI2, Cancer Metastasis Rev. 31 (3-4) (2012) 519-528.

[40] L.C. Fuller, et al., Expression of E-cadherin in human epidermal non-melanoma cutaneous tumours, Br. J. Dermatol. 134 (1) (1996) 28-32.

[41] J.R. Griffin, et al., Decreased expression of intercellular adhesion molecules in acantholytic squamous cell carcinoma compared with invasive well-differentiated squamous cell carcinoma of the skin, Am. J. Clin. Pathol. 139 (4) (2013) 442-447.

[42] A. Lyakhovitsky, et al., Expression of E-cadherin and beta-catenin in cutaneous squamous cell carcinoma and its precursors, Am. J. Dermatopathol. 26 (5) (2004) 372-378.
[43] I.H. Gelman, Emerging roles for SSeCKS/Gravin/AKAP12 in the control of cell proliferation, cancer malignancy, and barriergenesis, Genes Cancer 1 (11) (2010) 1147-1156

[44] W. Wu, et al., Examination of AKAP12 promoter methylation in skin cancer using methylation-sensitive high-resolution melting analysis, Clin. Exp. Dermatol. 36 (4) (2011) 381-385.

[45] C.-S. Yu, et al., Prediction of protein subcellular localization, Proteins: Struct. Funct. Bioinform. 64 (3) (2006) 643-651.

[46] A. Saha, E.S. Robertson, Functional modulation of the metastatic suppressor Nm23-H1 by oncogenic viruses, FEBS Lett. 585 (20) (2011) 3174-3184.

[47] N.I. Ismail, et al., Nuclear localization and intensity of staining of nm23 protein is useful marker for breast cancer progression, Cancer Cell Int. 8 (2008) p6.

[48] P. Lachat, et al., Expression of NDRG1, a differentiation-related gene, in human tissues, Histochem. Cell Biol. 118 (5) (2002) 399-408.

[49] A. Kawahara, et al., Nuclear expression of $\mathrm{N}$-myc downstream regulated gene $1 / \mathrm{Ca}(2+)$-associated protein 43 is closely correlated with tumor angiogenesis and poor survival in patients with gastric cancer, Exp. Ther. Med. 2 (3) (2011) 471-479.

[50] Y. Song, et al., Correlation of N-myc downstream-regulated gene 1 subcellular localization and lymph node metastases of colorectal neoplasms, Biochem. Biophys. Res. Commun. 439 (2) (2013) 241-246.

[51] D. Theodorescu, et al., Reduced expression of metastasis suppressor RhoGDI2 is associated with decreased survival for patients with bladder cancer, Clin. Cancer Res. 10 (11) (2004) 3800-3806. 